\title{
The Response of a Prestressed Beam-type Structure subjected to Partially Distributed Load Moving at Non- Uniform Velocities
}

\author{
Sunday T. Oni and Oluwatoyin K. Ogunbamike
}

\begin{abstract}
The transverse vibration of a prismatic Rayleigh beam resting on bi-parametric Vlasov foundation and continuously acted upon by partially distributed masses moving at varying velocities is investigated. For the solution of the fourth order partial differential equation with singular and variable coefficients, use is made of the technique based on the Generalized Finite Fourier Integral Transform, Struble's asymptotic technique and the use of Fresnel sine and cosine identities. Numerical results in plotted curves are presented. The results show that the response amplitude of the beam traversed by a distributed load moving with variable velocity decrease with an increase in the value of foundation modulus, Other structural parameters such as axial force, rotatory inertia and shear modulus are also found to reduce the displacement response of the beam as their values are increased in the dynamical system. The results also show that the critical speed for the system traversed by a moving distributed force is found to be greater than that traversed by moving mass. This confirms that the inertia effect of the moving distributed load must be considered for accurate and safe assessment of the response to moving distributed load of elastic structural members.
\end{abstract}

Index Terms-Continuously Acted; Distributed Load; Dynamical System; Vlasov Foundation.

\section{INTRODUCTION}

The flexural motion of Beam-type structures under moving loads is a very common problem in Engineering design and construction [1], [2]. The problem is usually modelled with the assumption that the load moves with constant velocity [3] which often is not realistic. When there is acceleration or deceleration in the mass motion over structures, the solution of moving mass problem becomes complicated and studies on this field are limited [4]- 6]. Worthy of note is the work of Huang and Thambiratnam [7]. They studied the deflection response of plate on Winkler foundation to moving accelerated load. The problem of the vibration of a prestressed non-uniform Rayleigh beam resting on elastic foundation and traversed by concentrated masses travelling at varying velocities was also investigated by Oni and Omolofe [8]. After this, the problem of flexural motions under accelerating loads of structurally prestressed beams with general boundary conditions was taken up by

Published on March 19, 2018.

S. T. Oni is with the Department of Mathematical Science, Federal University of Technology, Akure, NIGERIA (e-mail: sundayoni1958@yahoo.ca).

O. K. Ogunbamike is with the Department of Mathematical Sciences, Ondo State University of Science and Technology, Okitipupa, NIGERIA (e-mail: ogunbamike2005@gmail.com).
Oni and Omolofe [9]. It was concluded that higher values of pertinent structural parameters are required for a noticeable effect on the response of the beams in the case of other classical boundary conditions than those of simply supported end conditions for both moving force and moving mass problems. Recently, Alaa ([10] studied the simulation of transient dynamic load on beams with variable velocities. In particular, he studied the analysis and simulation of transient dynamic load on beam to find the effect of changing the initial velocity in the time-displacement amplitudes of the beam. It was concluded that changing initial velocity affect final displacement and time needed to reach it. Similarly, it was found that variance of final displacement generated between high velocities is small compared to final displacement generated between high and low velocities. Finite element method was used for the analysis of his results. Following closely is the work of Ismail [11] who investigated the problem of the response of a beam due to an accelerating moving mass using finite element approximation. It was concluded that acceleration of a travelling mass over a structural system highly affects the dynamic response of the structural system and that the inertia effects of the accelerating mass must be put into consideration in the model equation. The dynamical response of the rectangular plate resting on viscoelastic foundation and subjected to moving loads with variable velocity was taken up, very recently, by Mingliang, Tao, Yang Zhong and Hua Zhong [12]. The effects of the initial speed of the moving load and the damping of the foundation on the response are also discussed. Other researchers who have worked on the dynamic response of beam-type structures due to accelerated moving masses include Gbadeyan and Oni [13], Oni and Omolofe [14], Sun [15], Wang [16], Sadiku and Leipholz [17]. In all the aforementioned investigations, studies have been restricted to simplified load model often called concentrated loads. This consideration does not cover the wide range of application areas often encountered in Engineering practice. Emphatically speaking, an accurate and realistic representation of the moving load is that it is distributed over a small segment or over the entire length of the structure. This paper, therefore, investigates the dynamic analysis of a prestressed beam subjected to partially distributed load moving at varying velocities. Both gravity and inertial effects of the distributed load are taken into consideration and the beam is taken to rest on bi-parametric Vlasov foundation. The solution technique involves the use of generalized finite Fourier integral transform, the 
expression of the Heaviside in series form and Struble's asymptotic technique.

\section{MATHEMATICAL FORMULATION}

Consider the flexural motion of a prestressed finite uniform Rayleigh beam resting on bi-Parametric Vlasov foundation to a uniform partially distributed moving mass $M$ moving at non-uniform velocities. The Rayleigh beam has a constant cross sectional area and the mass $\mathbf{M}$ is assumed to touch the beam at time $\mathrm{t}=0$. The governing equation of motion with damping neglected is given by the fourth order partial differential equation.

$E I \frac{\partial^{4} V(x, t)}{\partial x^{4}}-N \frac{\partial^{2} V(x, t)}{\partial x^{2}}+\mu \frac{\partial^{2} V(x, t)}{\partial t^{2}}-\mu R^{0} \frac{\partial^{4} V(x, t)}{\partial x^{2} \partial t^{2}}+K V(x, t)-G \frac{\partial^{2} V(x, t)}{\partial x^{2}}=Q(x, t)$

where $E I$ is the flexural rigidity of the structure, $N$ is the axial force, $R^{0}$ is the rotatory inertia factor, $\mu$ is the mass per unit length of the beam, $V(x, t)$ is the transverse displacement, where $G$ is the shear modulus, $K$ is the foundation stiffness, $Q(x, t)$ is the transverse distributed load, $x$ is the spatial coordinate and $\mathrm{t}$ is the time. The load inertia is not negligible but significantly affects the behaviour of the dynamical system. Thus, the distributed load $Q(x, t)$ takes the form:

$Q(x, t)=\operatorname{MgH}[x-f(t)]\left[1-\frac{1}{g} \frac{d^{2} V(x, t)}{d t^{2}}\right]$

$g$ is the acceleration due to gravity and $H[x-f(t)]$ is the Heaviside function. Since the load is assumed to be of mass $M$ and the time $t$ is assumed to be limited to that interval of time within the mass on the beam, that is:

$$
0 \leq f(t) \leq L
$$

and $\frac{d^{2}}{d t^{2}}$ is a convective acceleration operator defined as

$$
\frac{d^{2}}{d t^{2}}=\frac{\partial^{2}}{\partial t^{2}}+\frac{2 d}{d t} f(t) \frac{\partial^{2}}{\partial x \partial t}+\left(\frac{d f(t)}{d t}\right)^{2} \frac{\partial^{2}}{\partial x^{2}}+\frac{d^{2}}{d t^{2}} f(t) \frac{\partial}{\partial x}
$$

The distance covered by the load on the same structure at any given instance of time is given as

$$
f(t)=x_{0}+c t+\frac{1}{2} a t^{2}
$$

where $x_{0}$ is the point of application of force at the instance $\mathrm{t}=0$, $\mathrm{c}$ is the initial velocity and $\mathrm{a}$ is the constant acceleration of motion. Using (2), (4) and (5) in (1), one obtains:
$E I \frac{\partial^{4} V(x, t)}{\partial x^{4}}-N \frac{\partial^{2} V(x, t)}{\partial x^{2}}+\mu \frac{\partial^{2} V(x, t)}{\partial t^{2}}-\mu R^{0} \frac{\partial^{4} V(x, t)}{\partial x^{2} \partial t^{2}}-G \frac{\partial^{2} V(x, t)}{\partial x^{2}}$

$+K V(x, t)+M H\left[x-\left(x_{0}+c t+\frac{1}{2} a t^{2}\right)\right]\left[\frac{\partial^{2} V(x, t)}{\partial t^{2}}+2(c+a t) \frac{\partial^{2} V(x, t)}{\partial x \partial t}\right.$

$\left.+\left(c+a t^{2}\right) \frac{\partial^{2} V(x, t)}{\partial x^{2}}+a \frac{\partial V(x, t)}{\partial x}\right]=M g H\left[x-\left(x_{0}+c t+\frac{1}{2} a t^{2}\right)\right]$

The boundary condition of the structure under consideration is taken to be simply supported. Thus, it is given by

$V(0, t)=V(L, t)=0 ; \quad=\frac{\partial^{2} V(0, t)}{\partial x^{2}}=\frac{\partial^{2} V(L, t)}{\partial x^{2}}$

The initial conditions without any loss of generality is taken as

$V(x, 0)=0=\frac{\partial V(x, 0)}{\partial t}$

Equation (6) is the fourth order partial differential equation with variable coefficients of the uniform Rayleigh beam under the action of travelling partially distributed loads moving with varying velocities.

In order to solve (6), evidently, the form of the boundary conditions (7) suggests the use of the finite Fourier sine transform defined by

\section{SOlution TECHNIQUES}

In order to solve (6), evidently, the form of the boundary conditions (7) suggests the use of the finite Fourier sine transform defined by

$\widetilde{V}(m, t)=\int_{0}^{L} V(x, t) U_{m}(x) d x$

with the inverse

$V(x, t)=\frac{1}{L} \sum_{m=1}^{\infty} \tilde{V}(m, t) \operatorname{Sin} \frac{m \pi x}{L}$

By applying the generalized finite integral transform (9), (6) becomes

$$
\begin{aligned}
& Z_{1} T_{A}(t)-Z_{2} T_{B}(t)+\widetilde{V}_{t t}(m, t)-R^{0} T_{C}(t)+Z_{3} T_{B}(t)+Z_{4} \tilde{V}(m, t) \\
& +T_{D}(t)+T_{E}(t)+T_{F}(t)+T_{G}(t)=\frac{M g}{\mu} T_{H}(t)
\end{aligned}
$$

Where

$$
Z_{1}=\frac{E I}{\mu}, Z_{2}=\frac{N}{\mu}, Z_{3}=\frac{G}{\mu}, Z_{4}=\frac{K}{\mu}
$$




$$
\begin{aligned}
& T_{A}(t)=\left(\frac{m \pi}{L}\right)_{0}^{4} \int_{0}^{L} \operatorname{Sin} \frac{m \pi x}{L} d x \\
& T_{B}(t)=\frac{1}{L} \sum_{k=1}^{\infty} \frac{\mu}{V_{k}} V(k, t) \frac{k^{2} \pi^{2}}{L} \int_{0}^{L} \operatorname{Sin} \frac{k \pi x}{L} \operatorname{Sin} \frac{m \pi x}{L} d x \\
& T_{C}(t)=\frac{\partial^{2}}{\partial t^{2}}\left\{\frac{1}{L} \sum_{k=1}^{\infty} \frac{\mu}{V_{k}} V(k, t) \frac{k^{2} \pi^{2} L}{L} \int_{0}^{L} \operatorname{Sin} \frac{k \pi x}{L} \operatorname{Sin} \frac{m \pi x}{L} d x\right\} \\
& T_{D}(t)=\frac{M}{\mu} \int_{0}^{L} H\left[x-\left(x_{0}+c t+\frac{1}{2} a t^{2}\right)\right] \frac{\partial^{2} V(x, t)}{\partial t^{2}} \operatorname{Sin} \frac{m \pi x}{L} d x(13 \mathrm{~d}) \\
& T_{E}(t)=\frac{2 M(c+a t)}{\mu} \int_{0}^{L} H\left[x-\left(x_{0}+c t+\frac{1}{2} a t^{2}\right)\right] \frac{\partial^{2} V(x, t)}{\partial x \partial t} \operatorname{Sin} \frac{m \pi x}{L} d x \\
& T_{F}(t)=\frac{2 M(c+a t)}{\mu} \int_{0}^{2} H\left[x-\left(x_{0}+c t+\frac{1}{2} a t^{2}\right)\right] \frac{\partial^{2} V(x, t)}{\partial x^{2}} \operatorname{Sin} \frac{m \pi x}{L} d x
\end{aligned}
$$

$T_{G}(t)=\frac{a M}{\mu} \int_{0}^{L} H\left[x-\left(x_{0}+c t+\frac{1}{2} a t^{2}\right)\right] \frac{\partial V(x, t)}{\partial x} \operatorname{Sin} \frac{m \pi x}{L} d x$

and

$$
T_{H}(t)=\int_{0}^{L} H\left[x-\left(x_{0}+c t+\frac{1}{2} a t^{2}\right)\right] \sin \frac{m \pi x}{L} d x
$$

In order to evaluate the integrals (13a) - (13h), use is made of the Fourier series representation for the Heaviside unit step function given as

$$
H\left[x-\left(x_{0}+c t+\frac{1}{2} a t^{2}\right)\right]=\frac{1}{4}+\frac{1}{\pi} \sum_{n=0}^{\infty} \frac{\operatorname{Sin}(2 n+1) \pi\left[x-\left(x_{0}+c t+\frac{1}{2} a t^{2}\right)\right]}{2 n+1},
$$

Adopting (14), integrals (13d) - (13g) can be simplify into

$$
\begin{aligned}
T_{D}(t) & =\sum_{k=1}^{\infty} \frac{M}{4 \mu V_{k}} \bar{V}_{t t}(k, t)\left[\int_{0}^{L} \operatorname{Sin} \frac{k \pi x}{L} \operatorname{Sin} \frac{m \pi x}{L} d x\right. \\
& +\frac{4}{\pi} \sum_{n=0}^{\infty} \frac{\operatorname{Cos}(2 n+1) \pi\left(x_{0}+c t+\frac{1}{2} a t^{2}\right)}{2 n+1} \int_{0}^{L} \operatorname{Sin} \frac{k \pi x}{L} \operatorname{Sin} \frac{m \pi x}{L}(x) d x \\
& \left.-\frac{4}{\pi} \sum_{n=0}^{\infty} \frac{\operatorname{Cos}(2 n+1) \pi\left(x_{0}+c t+\frac{1}{2} a t^{2}\right)_{L}^{L}}{2 n+1} \operatorname{Sin} \frac{k \pi x}{L} \operatorname{Sin} \frac{m \pi x}{L} d x\right]
\end{aligned}
$$

$$
\begin{aligned}
& T_{E}(t)=\sum_{k=1}^{\infty} \frac{M(c+a t)}{2 \mu V_{k}} \bar{V}_{t}(k, t) \frac{k \pi}{L}\left[\int_{0}^{L} \operatorname{Cos} \frac{k \pi x}{L} \operatorname{Sin} \frac{m \pi x}{L} d x\right. \\
& +\frac{4}{\pi} \sum_{n=0}^{\infty} \frac{\operatorname{Cos}(2 n+1) \pi\left(x_{0}+c t+\frac{1}{2} a t^{2}\right)}{2 n+1} \int_{0}^{L} \operatorname{Sin}(2 n+1) \pi x \operatorname{Cos} \frac{k \pi x}{L} \operatorname{Sin} \frac{m \pi x}{L} d x
\end{aligned}
$$

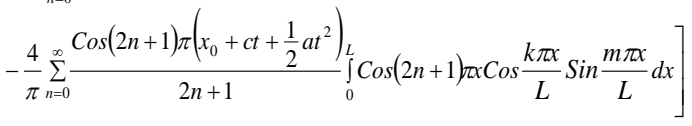

$$
\begin{aligned}
& T_{F}(t)=-\sum_{k=1}^{\infty} \frac{M(c+a t)^{2}}{4 \mu V_{k}} \bar{V}(k, t)\left(\frac{k \pi}{L}\right)^{2}\left[\int_{0}^{L} \operatorname{Sin} \frac{k \pi x}{L} \operatorname{Sin} \frac{m \pi x}{L} d x\right. \\
& +\frac{4}{\pi} \sum_{n=0}^{\infty} \frac{\operatorname{Cos}(2 n+1) \pi\left(x_{0}+c t+\frac{1}{2} a t^{2}\right)}{2 n+1} \int_{0}^{L} \operatorname{Sin} \frac{k \pi x}{L} \operatorname{Sin} \frac{m \pi x}{L}(x) d x \\
& \left.-\frac{4}{\pi} \sum_{n=0}^{\infty} \frac{\operatorname{Cos}(2 n+1) \pi\left(x_{0}+c t+\frac{1}{2} a t^{2}\right)_{L}}{2 n+1} \int_{0} \operatorname{Sin} \frac{k \pi x}{L} \operatorname{Sin} \frac{m \pi x}{L} d x\right] \\
& T_{G}(t)=\sum_{k=1}^{\infty} \frac{a M}{4 \mu V_{k}} \bar{V}(k, t) \frac{k \pi}{L}\left[\int_{0}^{L} \operatorname{Cos} \frac{k \pi x}{L} \operatorname{Sin} \frac{m \pi x}{L} d x\right. \\
& +\frac{4}{\pi} \sum_{n=0}^{\infty} \frac{\operatorname{Cos}(2 n+1) \pi\left(x_{0}+c t+\frac{1}{2} a t^{2}\right)_{L}}{2 n+1} \int_{0} \operatorname{Sin}(2 n+1) \pi x \operatorname{Cos} \frac{k \pi x}{L} \operatorname{Sin} \frac{m \pi x}{L} d x \\
& \left.-\frac{4}{\pi} \sum_{n=0}^{\infty} \frac{\operatorname{Cos}(2 n+1) \pi\left(x_{0}+c t+\frac{1}{2} a t^{2}\right)_{L}}{2 n+1} \int_{0} \operatorname{Cos}(2 n+1) z x \operatorname{Cos} \frac{k \pi x}{L} \operatorname{Sin} \frac{m \pi x}{L} d x\right]
\end{aligned}
$$

Substituting (13a)-(13c), (13h) and (15)-(18) with some simplifications and rearrangements, one obtains

$\tilde{V}_{t t}(m, t)+\left(\omega_{m}^{2}+\frac{K}{\mu}\right) \tilde{V}(m, t)-G \sum_{k=1}^{\infty} \tilde{V}(k, t) B_{A}(k, m)-R^{0} \sum_{k=1}^{\infty} \tilde{V}_{t t}(k, t) B_{A}(k, m)$

$-\frac{N}{\mu} \sum_{k=1}^{\infty} \tilde{V}(k, t) B_{A}(k, m)+\varepsilon_{0} L \sum_{k=1}^{\infty}\left[\left\{B_{B}(k, m)+\frac{1}{\pi} \sum_{n=0}^{\infty} \frac{\operatorname{Cos}(2 n+1) \pi\left(x_{0}+c t+\frac{1}{2} a t^{2}\right)}{2 n+1} B_{C}(n, k, m)\right.\right.$ $\left.-\frac{1}{\pi} \sum_{n=0}^{\infty} \frac{\operatorname{Sin}(2 n+1) \pi\left(x_{0}+c t+\frac{1}{2} a t^{2}\right)}{2 n+1} B_{D}(n, k, m)\right\} \tilde{V}_{t t}(k, t)+\left\{\frac{(c+a t)}{2} B_{E}(k, m)\right.$ $+\frac{2(c+a t)}{\pi} \sum_{n=0}^{\infty} \frac{\operatorname{Cos}(2 n+1) \pi\left(x_{0}+c t+\frac{1}{2} a t^{2}\right)}{2 n+1} B_{F}(n, k, m)$ $\left.-\frac{2(c+a t)}{\pi} \sum_{n=0}^{\infty} \frac{\operatorname{Sin}(2 n+1) \pi\left(x_{0}+c t+\frac{1}{2} a t^{2}\right)}{2 n+1} B_{G}(n, k, m)\right\} \tilde{V}_{t}(k, t)+\left\{\frac{(c+a t)^{2}}{4} B_{A}(k, m)\right.$ $+\frac{2(c+a t)^{2}}{\pi} \sum_{n=0}^{\infty} \frac{\operatorname{Cos}(2 n+1) \pi\left(x_{0}+c t+\frac{1}{2} a t^{2}\right)}{2 n+1} B_{H}(n, k, m)$

$-\frac{2(c+a t)^{2}}{\pi} \sum_{n=0}^{\infty} \frac{\operatorname{Sin}(2 n+1) \pi\left(x_{0}+c t+\frac{1}{2} a t^{2}\right)}{2 n+1} B_{I}(n, k, m)+\frac{a}{4} B_{E}(k, m)$

$\left.\left.\left.+\frac{a}{\pi} \sum_{n=0}^{\infty} \frac{\operatorname{Cos}(2 n+1) \pi\left(x_{0}+c t+\frac{1}{2} a t^{2}\right)}{2 n+1} B_{G}(n, k, m)-\frac{a}{\pi} \sum_{n=0}^{\infty} \frac{\operatorname{Sin}(2 n+1) \pi\left(x_{0}+c t+\frac{1}{2} a t^{2}\right)}{2 n+1} B_{H}(n, k, m)\right\} \tilde{V}(k, t)\right]\right\}$ $=\frac{P L}{m \pi \mu}\left[-\operatorname{Cos} m \pi+\operatorname{Cos} \frac{m \pi}{L}\left(x_{0}+c t+\frac{1}{2} a t^{2}\right)\right]$

where

$$
\varepsilon_{0}=\frac{M}{\mu L} ; \quad P=M g
$$

and 


$$
\begin{array}{ll}
B_{A}(k, m)=-\frac{k^{2} \pi^{2}}{V_{k} L^{2}} \int_{0}^{L} \operatorname{Sin} \frac{k \pi x}{L} \operatorname{Sin} \frac{m \pi x}{L} d x, & B_{B}(k, m)=\frac{1}{V_{k}} \int_{0}^{L} \operatorname{Sin} \frac{k \pi x}{L} \operatorname{Sin} \frac{m \pi x}{L} d x \\
B_{C}(k, m)=\frac{1}{V_{k}} \int_{0}^{L} \operatorname{Sin}(2 n+1) \pi x \operatorname{Sin} \frac{k \pi x}{L} \operatorname{Sin} \frac{m \pi x}{L} d x, & B_{D}(k, m)=\frac{1}{V_{k}} \int_{0}^{L} \operatorname{Cos}(2 n+1) \pi x \operatorname{Sin} \frac{k \pi x}{L} \operatorname{Sin} \frac{m \pi x}{L} d x \\
B_{E}(k, m)=\frac{k \pi}{V_{k} L} \int_{0}^{L} \operatorname{Cos} \frac{k \pi x}{L} \operatorname{Sin} \frac{m \pi x}{L} d x, & B_{F}(n, k, m)=\frac{k \pi}{V_{k} L} \int_{0}^{L} \operatorname{Sin}(2 n+1) \pi x \operatorname{Cos} \frac{k \pi x}{L} \operatorname{Sin} \frac{m \pi x}{L} d x \\
B_{G}(k, m)=\frac{k \pi}{V_{k} L} \int_{0}^{L} \operatorname{Cos}(2 n+1) \pi x \operatorname{Cos} \frac{k \pi x}{L} \operatorname{Sin} \frac{m \pi x}{L} d x, & B_{H}(k, m)=-\frac{k^{2} \pi^{2}}{V_{k} L^{2}} \int_{0}^{L} \operatorname{Sin}(2 n+1) \pi x \operatorname{Sin} \frac{k \pi x}{L} \operatorname{Sin} \frac{m \pi x}{L} d x \\
B_{I}(k, m)=\frac{k^{2} \pi^{2}}{V_{k} L^{2}} \int_{0}^{L} \operatorname{Cos}(2 n+1) \pi x \operatorname{Sin} \frac{k \pi x}{L} \operatorname{Sin} \frac{m \pi x}{L} d x, & V_{K}=\int_{0}^{L} \operatorname{Sin} \frac{k \pi x}{L} \operatorname{Sin} \frac{m \pi x}{L} d x
\end{array}
$$

Solving integrals in (21) and substituting into (19) yields

$$
\begin{aligned}
& \left(1+R^{0} \frac{m^{2} \pi^{2}}{L^{2}}\right) \tilde{V}_{t t}(m, t)+\left[\frac{E I}{\mu}\left(\frac{m \pi}{L}\right)^{4}+\frac{K}{\mu}+\frac{(N+G)}{\mu}\left(\frac{m \pi}{L}\right)^{2}\right] \tilde{V}(m, t)+\varepsilon_{0} L\left\{\left(\frac{1}{4}\right.\right. \\
& +\frac{L}{\pi^{2}} \sum_{n=0}^{\infty}(2 n+1) R_{a}(k, m) \frac{\operatorname{Cos}(2 n+1) \pi\left(x_{0}+c t+\frac{1}{2} a t^{2}\right)}{2 n+1} \\
& \left.-\frac{L}{\pi^{2}} \sum_{n=0}^{\infty}(2 n+1) R_{b}(k, m) \frac{\operatorname{Sin}(2 n+1) \pi\left(x_{0}+c t+\frac{1}{2} a t^{2}\right)}{2 n+1}\right) \tilde{V}_{t t}(m, t) \\
& +\frac{2 m(c+a t)}{\pi L}\left(\sum_{n=0}^{\infty} R_{c}(k, m) \frac{\operatorname{Cos}(2 n+1) \pi\left(x_{0}+c t+\frac{1}{2} a t^{2}\right)}{2 n+1}-\sum_{n=0}^{\infty} R_{d}(k, m) \frac{\operatorname{Sin}(2 n+1) \pi\left(x_{0}+c t+\frac{1}{2} a t^{2}\right)}{2 n+1}\right) \tilde{V}_{t}(m, t) \\
& +\left[\frac { ( c + a t ) m ^ { 2 } \pi ^ { 2 } } { L ^ { 2 } } \left(\frac{1}{4}+\frac{L}{\pi^{2}} \sum_{n=0}^{\infty}(2 n+1) R_{a}(k, m) \frac{\operatorname{Cos}(2 n+1) \pi\left(x_{0}+c t+\frac{1}{2} a t^{2}\right)}{2 n+1}\right.\right. \\
& \left.-\frac{L}{\pi^{2}} \sum_{n=0}^{\infty}(2 n+1) R_{b}(k, m) \frac{\operatorname{Sin}(2 n+1) \pi\left(x_{0}+c t+\frac{1}{2} a t^{2}\right)}{2 n+1}\right)-\frac{a m}{L \pi}\left(\sum_{n=0}^{\infty} R_{c}(k, m) \frac{\operatorname{Cos}(2 n+1) \pi\left(x_{0}+c t+\frac{1}{2} a t^{2}\right)}{2 n+1}\right. \\
& \left.\left.\left.\sum_{n=0}^{\infty} R_{d}(k, m) \frac{\operatorname{Sin}(2 n+1) \pi\left(x_{0}+c t+\frac{1}{2} a t^{2}\right)}{2 n+1}\right)\right] \tilde{V}(m, t)\right\} \\
& +\varepsilon_{0} L\left\{\sum _ { \substack { k = 1 \\
k \neq m } } ^ { \infty } \left(\frac{L}{\pi^{2}} \sum_{n=0}^{\infty}(2 n+1) R_{e}(k, m) \frac{\operatorname{Cos}(2 n+1) \pi\left(x_{0}+c t+\frac{1}{2} a t^{2}\right)}{2 n+1}-\frac{L}{\pi^{2}} \sum_{n=0}^{\infty}(2 n+1) R_{f}(k, m)\right.\right. \\
& \left.\frac{\operatorname{Sin}(2 n+1) \pi\left(x_{0}+c t+\frac{1}{2} a t^{2}\right)}{2 n+1}\right) \tilde{V}_{t t}(k, t)+\frac{2 k \pi(c+a t)}{L}\left(\frac{1}{\pi^{2}} \sum_{n=0}^{\infty} N_{g}(k, m) \frac{\operatorname{Cos}(2 n+1) \pi\left(x_{0}+c t+\frac{1}{2} a t^{2}\right)}{2 n+1}\right. \\
& -\frac{1}{\pi^{2}} \sum_{n=0}^{\infty} R_{h}(k, m) \cdot \frac{\operatorname{Sin}(2 n+1) \pi\left(x_{0}+c t+\frac{1}{2} a t^{2}\right)}{2 n+1} \tilde{V}_{t}(k, t) \\
& +\left[\frac { ( c + a t ) ^ { 2 } k ^ { 2 } \pi ^ { 2 } } { L ^ { 2 } } \left(\frac{L}{\pi^{2}} \sum_{n=0}^{\infty} R_{e}(k, m) \frac{\operatorname{Cos}(2 n+1) \pi\left(x_{0}+c t+\frac{1}{2} a t^{2}\right)}{2 n+1}\right.\right. \\
& \left.-\frac{L}{\pi^{2}} \sum_{n=0}^{\infty}(2 n+1) R_{f}(k, m) \frac{\operatorname{Sin}(2 n+1) \pi\left(x_{0}+c t+\frac{1}{2} a t^{2}\right)}{2 n+1}\right)-\frac{a k \pi}{L}\left(\frac { 1 } { \pi ^ { 2 } } \left(\sum_{n=0}^{\infty} R_{g}(k, m) \frac{\operatorname{Cos}(2 n+1) \pi\left(x_{0}+c t+\frac{1}{2} a t^{2}\right)}{2 n+1}\right.\right. \\
& \left.\left.\left.-\frac{1}{\pi^{2}} \sum_{n=0}^{\infty} R_{h}(k, m) \frac{\operatorname{Sin}(2 n+1) \pi\left(x_{0}+c t+\frac{1}{2} a t^{2}\right)}{2 n+1}\right)\right] \tilde{V}(k, t)\right\}=\frac{P L}{\mu m \pi}\left[-\operatorname{Cos} m \pi+\operatorname{Cos} \frac{m \pi}{L}\left(x_{0}+c t+\frac{1}{2} a t^{2}\right)\right]
\end{aligned}
$$

where

$$
\begin{aligned}
& R_{e}(k, m)=\left[\frac{(-1)^{k+m} \operatorname{Cos}(2 n+1) \pi L-1}{[(2 n+1) L]^{2}-(k+m)^{2}}-\frac{(-1)^{k-m} \operatorname{Cos}(2 n+1) \pi L-1}{[(2 n+1) L]^{2}-(k-m)^{2}}\right] \\
& R_{b}(k, m)=\left[\frac{1}{[(2 n+1) L]^{2}}-\frac{(-1)^{2 m}}{[(2 n+1) L]^{2}-4 m^{2}}\right] \operatorname{Sin}(2 n+1) \pi L \\
& R_{c}(k, m)=\left[\frac{2 m(-1)^{2 m} \operatorname{Sin}(2 n+1) \pi L}{[(2 n+1) L]^{2}-4 m^{2}}\right] ; \quad R_{d}(k, m)=\left[\frac{2 m\left\{(-1)^{2 m} \operatorname{Cos}(2 n+1) \pi L-1\right\}}{[(2 n+1) L]^{2}-4 m^{2}}\right] \\
& R_{f}(k, m)=\left[\frac{(-1)^{k+m}}{[(2 n+1) L]^{2}-(k+m)^{2}}-\frac{(-1)^{k-m}}{[(2 n+1) L]^{2}-(k-m)^{2}}\right] \operatorname{Sin}(2 n+1) \pi L \\
& R_{g}(k, m)=\left[\frac{(k+m)(-1)^{(k+m)} \operatorname{Sin}(2 n+1) \pi L}{[(2 n+1) L]^{2}-(k+m)^{2}}-\frac{(k-m)(-1)^{(k-m)} \operatorname{Sin}(2 n+1) \pi L}{[(2 n+1) L]^{2}-(k-m)^{2}}\right] \\
& R_{h}(k, m)=\left[\frac{(k+m)\left\{(-1)^{(k+m)} \operatorname{Cos}(2 n+1) \pi L-1\right\}}{[(2 n+1) L]^{2}-(k+m)^{2}}-\frac{(k-m)\left\{(-1)^{(k-m)} \operatorname{Cos}(2 n+1) \pi L-1\right\}}{[(2 n+1) L]^{2}-(k-m)^{2}}\right]
\end{aligned}
$$

Equation (22) is the fundamental equation of our problem when the Rayleigh beam has simple supports at all edges. In what follows, we shall discuss two cases of the equation.

\section{A. Case I: Uniform Rayleigh Beam Traversed by Moving Partially Distributed Force}

This approximate model of the system is obtained when the inertia effect of the moving distributed mass $M$ is neglected. Thus by setting $\varepsilon_{0}$ to zero in (22), yields

$$
\begin{aligned}
& \left(1+R^{0} \frac{m^{2} \pi^{2}}{L^{2}}\right) \tilde{V}_{t t}(m, t)+\left[\frac{E I}{\mu}\left(\frac{m \pi}{L}\right)^{4}+\frac{K}{\mu}+\frac{(N+G)}{\mu}\left(\frac{m \pi}{L}\right)^{2}\right] \tilde{V}(m, t) \\
& =\frac{P L}{\mu m \pi}\left[-\operatorname{Cos} m \pi+\operatorname{Cos} \frac{m \pi}{L}\left(x_{0}+c t+\frac{1}{2} a t^{2}\right)\right]
\end{aligned}
$$

which when rearranged gives

$$
\bar{V}_{t t}(m, t)+\gamma_{p p}^{2} \bar{V}(m, t)=P_{p f}\left[-(-1)^{m}+\cos \frac{m \pi}{L}\left(x_{0}+c t+\frac{1}{2} a t^{2}\right]\right.
$$

where

$$
\gamma_{p p}^{2}=\frac{\left[\frac{E I}{\mu}\left(\frac{m \pi}{L}\right)^{4}+\frac{K}{\mu}+\frac{(N+G)}{\mu}\left(\frac{m \pi}{L}\right)^{2}\right]}{1+R^{0}\left(\frac{m \pi}{L}\right)^{2}}, P_{p f}=\frac{P L}{\mu m \pi\left[1+R^{0}\left(\frac{m \pi}{L}\right)^{2}\right]}
$$

Solving (25) using the method of variation of parameters technique in conjunction with the initial conditions gives the expression for $\tilde{V}(m, t)$ which on inversion yields

$$
\begin{aligned}
& \bar{V}(x, t)=\frac{2}{L} \sum_{m=12 \gamma}^{\infty} \frac{P_{p f} \sqrt{\pi}}{2 \gamma_{p} \sqrt{2 a}}\left\{\operatorname { S i n \gamma } p p ^ { t } \left[\operatorname{Sin}\left(\frac{b_{2}^{2}}{4 a}-c_{0}\right) s\left(\frac{b_{2}+2 a t}{\sqrt{2 \pi a}}\right)+\operatorname{Cos}\left(\frac{b_{2}^{2}}{4 a}-c_{0}\right) C\left(\frac{b_{2}+2 a t}{\sqrt{2 \pi u}}\right)\right.\right. \\
& +\operatorname{Sin}\left(\frac{b^{2}}{4 a}-c_{0}\right) S\left(\frac{b_{1}+2 a t}{\sqrt{2 \pi a}}\right)+\operatorname{Cos}\left(\frac{b_{1}^{2}}{4 a}-c_{0}\right) c\left(\frac{b_{1}+2 a t}{\sqrt{2 \pi a}}\right)-\operatorname{Sin}\left(\frac{b_{2}^{2}}{4 a}-c_{0}\right) S\left(\frac{b_{2}}{\sqrt{2 \pi a}}\right)-\operatorname{Cos}\left(\frac{b_{2}^{2}}{4 a}-c_{0}\right) c\left(\frac{b_{2}}{\sqrt{2 \pi a}}\right) \\
& -\operatorname{Sin}\left(\frac{b_{1}^{2}}{4 a}-c_{0}\right) S\left(\frac{b_{1}}{\sqrt{2 \pi a}}\right)-\operatorname{Cos}\left(\frac{b^{2}}{4 a}-c_{0}\right) c\left(\frac{b_{1}}{\sqrt{2 \pi a}}\right)-\frac{1}{2 \gamma}\left[\operatorname{Sin}\left(m \pi+\gamma_{p p} t\right)-\operatorname{Sin}\left(m \pi-\gamma_{p p} t\right)\right] \\
& -\frac{\operatorname{Cos} \gamma p p^{t}}{\gamma_{p p}}\left[-\operatorname{Sin}\left(\frac{b^{2}}{4 a}-c_{0}\right) c\left(\frac{b_{1}+2 a t}{\sqrt{2 \pi a}}\right)+\operatorname{Cos}\left(\frac{b_{1}^{2}}{4 a}-c_{0}\right) S\left(\frac{b_{1}+2 a t}{\sqrt{2 \pi a}}\right)+\operatorname{Sin}\left(\frac{b_{2}^{2}}{4 a}-c_{0}\right) c\left(\frac{b_{2}+2 a t}{\sqrt{2 \pi a}}\right)\right. \\
& -\operatorname{Cos}\left(\frac{b_{2}^{2}}{4 a}-c_{0}\right) S\left(\frac{b_{2}+2 a t}{\sqrt{2 \pi a}}\right)+\operatorname{Sin}\left(\frac{b_{1}^{2}}{4 a}-c_{0}\right) c\left(\frac{b_{1}}{\sqrt{2 \pi a}}\right)-\operatorname{Cos}\left(\frac{b_{1}^{2}}{4 a}-c_{0}\right) S\left(\frac{b_{1}}{\sqrt{2 \pi a}}\right)-\operatorname{Sin}\left(\frac{b_{2}^{2}}{4 a}-c_{0}\right) c\left(\frac{b_{2}}{\sqrt{2 \pi a}}\right) \\
& \left.+\operatorname{Cos}\left(\frac{b_{2}^{2}}{4 a}-c_{0}\right) S\left(\frac{b_{2}}{\sqrt{2 \pi a}}\right)+\frac{\operatorname{Cos} m \pi}{\gamma_{p p}}+\frac{1}{2 \gamma_{p p}}\left[\operatorname{Cos}\left(m \pi-\gamma_{p p} t\right)+\operatorname{Cos}\left(m \pi+\gamma_{p p} t\right)\right]\right\}\left(\operatorname{Sin} \frac{\mathrm{m} \pi}{\mathrm{L}} x\right)
\end{aligned}
$$

where $C(x)$ and $S(x)$ are the well-known time-dependent Fresnel integrals defined by

$$
C(x)=\int_{0}^{x} \cos \frac{\pi t^{2}}{2} d t ; \quad S(x)=\int_{0}^{x} \sin \frac{\pi t^{2}}{2} d t
$$

Equation (27) represents the transverse displacement response to a partially distributed force moving at nonuniform velocity of a simply supported uniform Rayleigh beam resting on bi-parametric Pasternak foundation. 


\section{B. Case II: Uniform Rayleigh Beam Traversed by Moving Partially Distributed Mass}

If the moving load has mass commensurable with that of the elastic beam, the inertia effect of the moving mass is not negligible and must be taken into consideration. Thus, $\varepsilon_{0} \neq 0$ and we are required to solve the entire (22) Evidently, an exact closed form solution of this equation is not possible. Thus we resort to the approximate analytical solution technique which is a modification of the asymptotic method of Struble discussed in [15]. To this end, equation (22) is rearranged to take the form

$$
\begin{aligned}
& \tilde{V}_{t t}(m, t)+\frac{2 m(c+a t) Y_{b}(m, n, t)}{\pi Q_{A}\left(1+\frac{\varepsilon_{0} L}{Q_{A}} Y_{a}(m, n, t)\right.} \tilde{V} t(m, t)+\left(\frac{\frac{(c+a t)^{2} m^{2} \pi^{2} \varepsilon_{0} Y_{a}(m, n, t)}{Q_{A} L}-\frac{a m \varepsilon_{0} Y_{b}(m, n, t)+\gamma_{p p}^{2}}{Q_{A} \pi}}{1+\frac{\varepsilon_{0} L}{Q_{A}} Y_{a}(m, n, t)}\right) \tilde{V}(m, t) \\
& +\frac{\varepsilon_{0} L}{Q_{A}\left(1+\frac{\varepsilon_{0} L}{Q_{A}} Y_{a}(m, n, t)\right.}\left\{\sum _ { \substack { k = 1 \\
k \neq m } } ^ { \infty } \left(\frac{L}{\pi^{2}} \sum_{n=0}^{\infty}(2 n+1) R_{e}(k, m) \frac{\operatorname{Cos}(2 n+1) \pi\left(x_{0}+c t+\frac{1}{2} a t^{2}\right)}{2 n+1}\right.\right. \\
& \left.-\frac{L}{\pi^{2}} \sum_{n=0}^{\infty}(2 n+1) R_{f}(k, m) \frac{\operatorname{Sin}(2 n+1) \pi\left(x_{0}+c t+\frac{1}{2} a t^{2}\right)}{2 n+1}\right) \tilde{V}_{t t}(k, t) \\
& +\frac{2(c+a t) k \pi}{L}\left(\frac{1}{\pi^{2}} \sum_{n=0}^{\infty} R_{g}(k, m) \frac{\operatorname{Cos}(2 n+1) \pi\left(x_{0}+c t+\frac{1}{2} a t^{2}\right)}{2 n+1}\right. \\
& \left.-\frac{1}{\pi^{2}} \sum_{n=0}^{\infty} R_{h}(k, m) \frac{\operatorname{Sin}(2 n+1) \pi\left(x_{0}+c t+\frac{1}{2} a t^{2}\right)}{2 n+1}\right) \tilde{V}_{t}(k, t) \\
& +\left[\frac { ( c + a t ) ^ { 2 } k ^ { 2 } \pi ^ { 2 } } { L ^ { 2 } } \left(\frac{1}{4}+\frac{L}{\pi^{2}} \sum_{n=0}^{\infty}(2 n+1) R_{e}(k, m) \frac{\operatorname{Cos}(2 n+1) \pi\left(x_{0}+c t+\frac{1}{2} a t^{2}\right)}{2 n+1}\right.\right. \\
& \left.+\frac{1}{\pi^{2}} \sum_{n=0}^{\infty}(2 n+1) R_{f}(k, m) \frac{\operatorname{Sin}(2 n+1) \pi\left(x_{0}+c t+\frac{1}{2} a t^{2}\right)}{2 n+1}\right) \\
& -\frac{a \pi k}{L}\left(\frac{1}{\pi^{2}} \sum_{n=0}^{\infty}(2 n+1) R_{g}(k, m) \frac{\operatorname{Cos}(2 n+1) \pi\left(x_{0}+c t+\frac{1}{2} a t^{2}\right)}{2 n+1}\right. \\
& \left.\left.-\frac{1}{\pi^{2}} \sum_{n=0}^{\infty}(2 n+1) R_{h}(k, m) \frac{\operatorname{Sin}(2 n+1) \pi\left(x_{0}+c t+\frac{1}{2} a t^{2}\right)}{2 n+1}\right) \tilde{V}(k, t)\right\} \\
& =\frac{P L}{\mu m \pi Q_{A}\left(+\frac{\varepsilon_{0} L}{Q_{A}} Y_{I}(m, n, t)\right)}\left[-(-1)^{m}+\operatorname{Cos} \frac{m \pi}{L}\left(x_{0}+c t+\frac{1}{2} a t^{2}\right)\right]
\end{aligned}
$$

where

$$
\begin{gathered}
Q_{A}=1+R^{0}\left(\frac{m \pi}{L}\right)^{2} \\
Y_{a}(m . n . t)=\frac{1}{4}+\frac{L}{\pi^{2}} \sum_{n=0}^{\infty}(2 n+1) R_{a}(k, m) \frac{\operatorname{Cos}(2 n+1) \pi\left(x_{0}+c t+\frac{1}{2} a t^{2}\right)}{2 n+1} \\
\left.-\frac{L}{\pi^{2}} \sum_{n=0}^{\infty}(2 n+1) R_{b}(k, m) \frac{\operatorname{Sin}(2 n+1) \pi\left(x_{0}+c t+\frac{1}{2} a t^{2}\right)}{2 n+1}\right) \\
Y_{b}(m . n . t)= \\
\quad \sum_{n=0}^{\infty} R_{c}(k, m) \frac{\operatorname{Cos}(2 n+1) \pi\left(x_{0}+c t+\frac{1}{2} a t^{2}\right)}{2 n+1} \\
-\sum_{n=0}^{\infty} R_{d}(k, m) \frac{\operatorname{Sin}(2 n+1) \pi\left(x_{0}+c t+\frac{1}{2} a t^{2}\right)}{2 n+1}
\end{gathered}
$$

By this method, we seek the modified frequency corresponding to the frequency of the free system due to the presence of the moving mass. An equivalent free system operator defined by the modified frequency then replaces (29). To this end, the right hand side of (29) is set to zero and a parameter $\Gamma_{1}<1$ is considered for any arbitrary mass ratio $\varepsilon_{0}$ defined as

$$
\Gamma_{1}=\frac{\varepsilon_{0}}{1+\varepsilon_{0}}
$$

It can easily be shown that

$$
\varepsilon_{0}=\Gamma_{1}+O\left(\Gamma_{1}^{2}\right)
$$

which implies

$$
\frac{1}{\left(1+\frac{\varepsilon_{0} L}{Q_{A}} Y_{I}(m, n, t)\right.}=\left(1-\frac{\Gamma_{1} L}{Q_{A}} Y_{I}(m, n, t)+O\left(\Gamma_{1}^{2}\right)\right.
$$

And

$$
\mid 1+\frac{\Gamma_{1} L}{Q_{A}} Y_{I}(m, n, t \mid<1
$$

When $\Gamma_{1}$ is set to zero in (29), a case corresponding to the case when the inertia effect of the mass of the system is neglected is obtained and the solution of (29) can be written in the form

$$
\bar{V}(m, t)=A_{0} \operatorname{Cos}\left[\gamma_{p p} t-\phi_{m}\right]
$$

where $\gamma_{p p}$ is as previously defined, $A_{0}$ and $\phi_{m}$ are constants. Since $\Gamma_{1}<1$, Struble's technique requires that the asymptotic solution of the homogeneous part of (31) can be written as

$$
\bar{V}(m, t)=\nabla(m, t) \operatorname{Cos}\left[\gamma_{p p} t-\phi(m, t)\right]+\Gamma_{1} \bar{V}(m, t)+O\left(\Gamma_{1}^{2}\right)
$$

where $\nabla(m, t)$ and $\phi(m, t)$ are slowly varying functions of time.

Substituting (38) and its derivatives into the homogeneous part of (31) and taking into account (34) and retaining terms to $O\left(\Gamma_{1}\right)$, one obtains 
$-2 \dot{\nabla}(m, t) \gamma_{p p} \operatorname{Sin}\left[\gamma_{p p} t-\phi(m, t)\right]+2 \nabla(m, t) \dot{\phi}(m, t) \gamma_{p p} \operatorname{Cos}\left[\gamma_{p p} t-\phi(m, t)\right]$

$+\left(\frac{(c+a t)^{2} m^{2} \pi \Gamma_{1} Y_{I}(m, n, t)}{Q_{A}{ }^{2}}-\frac{a m \Gamma_{1} Y_{I I}(m, n, t)+\gamma_{p p}^{2}}{Q_{A} \pi}-\frac{\gamma \gamma_{p p}^{2} \Gamma_{1} Y_{I}(m, n, t)}{Q_{A}}\right)$.

$\nabla(m, t) \operatorname{Cos}\left[\gamma_{p p} t-\phi(m, t)\right]+\frac{\Gamma_{1} L}{Q_{A}}\left\{\sum_{\substack{k=1 \\ k \neq m}}^{\infty}\left(\frac{L}{\pi^{2}}(2 n+1) R_{e}(n, k, m) \frac{\operatorname{Cos}(2 n+1) \pi\left(x_{0}+c t+\frac{1}{2} a t^{2}\right)}{2 n+1}\right.\right.$

$+\frac{1}{\pi} \sum_{n=0}^{\infty}(2 n+1) R_{f}\left(n, k, m \frac{\operatorname{Sin}(2 n+1) \pi\left(x_{0}+c t+\frac{1}{2} a t^{2}\right)}{2 n+1}\right) H_{11}(k, t)+$

$+\frac{2(c+a t) k \pi}{L}\left(\frac{1}{\pi^{2}} \sum_{n=0}^{\infty} R_{g}(k, m) \frac{\operatorname{Cos}(2 n+1) \pi\left(x_{0}+c t+\frac{1}{2} a t^{2}\right)}{2 n+1}\right.$

$\left.-\frac{1}{\pi^{2}} \sum_{n=0}^{\infty} R_{h}(k, m) \frac{\operatorname{Sin}(2 n+1) \pi\left(x_{0}+c t+\frac{1}{2} a t^{2}\right)}{2 n+1}\right) H_{12}(k, t)$

$+\left[\frac{(c+a t)^{2} k^{2} \pi^{2}}{L^{2}}\left(\frac{L}{\pi^{2}} \sum_{n=0}^{\infty}(2 n+1) N_{5}(k, m) \frac{\operatorname{Cos}(2 n+1) \pi\left(x_{0}+c t+\frac{1}{2} a t^{2}\right)}{2 n+1}\right.\right.$

$\left.+\frac{L}{\pi^{2}} \sum_{n=0}^{\infty}(2 n+1) R_{f}(k, m) \frac{\operatorname{Sin}(2 n+1) \pi\left(x_{0}+c t+\frac{1}{2} a t^{2}\right)}{2 n+1}\right)$

$-\frac{a \pi k}{L}\left(\frac{1}{\pi^{2}} \sum_{n=0}^{\infty} R_{g}(k, m) \frac{\operatorname{Cos}(2 n+1) \pi\left(x_{0}+c t+\frac{1}{2} a t^{2}\right)}{2 n+1}\right.$

$\left.\left.-\frac{1}{\pi^{2}} \sum_{n=0}^{\infty} R_{h}(k, m) \frac{\operatorname{Sin}(2 n+1) \pi\left(x_{0}+c t+\frac{1}{2} a t^{2}\right)}{2 n+1}\right) H_{13}(k, t)\right\}=0$

where

$$
\begin{aligned}
H_{11}(k, t)= & \left.2 \dot{\nabla}(k, t) \gamma_{p p} \operatorname{Sin} \mid \gamma_{p p} t-\phi(k, t)\right] \\
& +2 \nabla(k, t) \dot{\phi}(k, t) \gamma_{p p} \operatorname{Cos}\left[\gamma_{p p} t-\phi(k, t)\right]-\nabla(k, t) \gamma_{p p}^{2} \operatorname{Cos}\left[\gamma_{p p} t-\phi(k, t)\right] \\
H_{12}(k, t)= & \dot{\nabla}(k, t) \operatorname{Cos}\left[\gamma_{p p} t-\phi(k, t)\right]+\nabla(k, t) \dot{\phi}(k, t) \operatorname{Sin}\left[\gamma_{p p} t-\phi(k, t)\right] \\
& +\nabla(k, t) \gamma_{p p} \operatorname{Sin}\left[\gamma_{p p} t-\phi(k, t)\right] \\
H_{13}(k, t)= & \nabla(k, t) \operatorname{Cos}\left[\gamma_{p p} t-\phi(k, t)\right]
\end{aligned}
$$

Next, we extract only the variational part of the equation describing the behaviour of $\nabla(m, t)$ and $\phi(m, t)$ during the motion of the distributed mass. To this end, we take into account the following trigonometric identities

$$
\begin{aligned}
\frac{\operatorname{Cos}(2 n+1) \pi\left(x_{0}+c t+\frac{1}{2} a t^{2}\right)}{2 n+1} \operatorname{Cos}\left[\gamma_{p p} t-\phi(m, t)\right] & =\frac{1}{2} \operatorname{Cos}\left[\frac{(2 n+1) \pi\left(x_{0}+c t+\frac{1}{2} a t^{2}\right)}{2 n+1}+\gamma_{p p} t-\phi(m, t)\right] \\
& +\frac{1}{2} \operatorname{Cos}\left[\frac{(2 n+1) \pi\left(x_{0}+c t+\frac{1}{2} a t^{2}\right)}{2 n+1}-\gamma_{p p} t-\phi(m, t)\right]
\end{aligned}
$$

$$
\begin{aligned}
\frac{\operatorname{Cos}(2 n+1) \pi\left(x_{0}+c t+\frac{1}{2} a t^{2}\right)}{2 n+1} \operatorname{Cos}\left[\gamma_{p p} t-\phi(m, t)\right] & =\frac{1}{2} \operatorname{Cos}\left[\frac{(2 n+1) \pi\left(x_{0}+c t+\frac{1}{2} a t^{2}\right)}{2 n+1}+\gamma_{p p} t-\phi(m, t)\right] \\
& +\frac{1}{2} \operatorname{Cos}\left[\frac{(2 n+1) \pi\left(x_{0}+c t+\frac{1}{2} a t^{2}\right)}{2 n+1}-\gamma_{p p} t-\phi(m, t)\right]
\end{aligned}
$$

$$
\begin{aligned}
\frac{\operatorname{Cos}(2 n+1) \pi\left(x_{0}+c t+\frac{1}{2} a t^{2}\right)}{2 n+1} \operatorname{Sin}\left[\gamma_{p p} t-\varphi(m, t)\right] & =\frac{1}{2} \operatorname{Sin}\left[\frac{(2 n+1) \pi\left(x_{0}+c t+\frac{1}{2} a t^{2}\right)}{2 n+1}+\gamma_{p p} t-\phi(m, t)\right] \\
& -\frac{1}{2} \operatorname{Sin}\left[\frac{(2 n+1) \pi\left(x_{0}+c t+\frac{1}{2} a t^{2}\right)}{2 n+1}-\gamma_{p p} t+\phi(m, t)\right]
\end{aligned}
$$

$$
\begin{aligned}
\frac{\operatorname{Sin}(2 n+1) \pi\left(x_{0}+c t+\frac{1}{2} a t^{2}\right)}{2 n+1} \operatorname{Sin}\left[\gamma_{p p} t-\phi(m, t)\right]= & \frac{1}{2} \operatorname{Cos}\left[\frac{(2 n+1) \pi\left(x_{0}+c t+\frac{1}{2} a t^{2}\right)}{2 n+1}-\gamma_{p p} t+\phi(m, t)\right] \\
& -\frac{1}{2} \operatorname{Cos}\left[\frac{(2 n+1) \pi\left(x_{0}+c t+\frac{1}{2} a t^{2}\right)}{2 n+1}+\gamma_{p p} t-\phi(m, t)\right]
\end{aligned}
$$

and neglecting terms that do not contribute to the variational equation, equation (40) reduces

$$
\begin{aligned}
& \left.-2 \dot{\nabla}(m, t) \gamma_{p p} \operatorname{Sin} \mid \gamma_{p p} t-\phi(m, t)\right\rfloor+2 \nabla(m, t) \dot{\phi}(m, t) \gamma_{p p} \operatorname{Cos}\left[\gamma_{p p} t-\phi(m, t)\right] \\
& +\frac{c^{2} m^{2} \pi \Gamma_{1}}{4 Q_{A} L} \nabla(m, t) \operatorname{Cos}\left[\gamma_{p p} t-\phi(m, t)\right]-\frac{\gamma_{p p}^{2} \Gamma_{1} L}{Q_{A}} \nabla(m, t) \operatorname{Cos}\left[\gamma_{p p} t-\phi(m, t)\right]=0
\end{aligned}
$$

The variational equations of our problem are obtained by setting the coefficients of $\operatorname{Cos}\left\lfloor\gamma_{p p} t-\phi(m, t)\right\rfloor$ and $\operatorname{Sin}\left[\gamma_{p p} t-\phi(m, t)\right]$ in equation (39) to zero respectively. Thus we have

$$
-2 \dot{\nabla}(m, t) \gamma_{p p}=0
$$

and

$2 \nabla(m, t) \dot{\phi}(m, t) \gamma_{p p}+\frac{c^{2} m^{2} \pi \Gamma_{1}}{4 Q_{A} L} \nabla(m, t)-\frac{\gamma_{p p}^{2} \Gamma_{1} L}{Q_{A}} \nabla(m, t)=0$

Solving (46) and (47) respectively, one obtains

$$
\nabla(m, t)=D_{m}
$$

and

$$
\phi(m, t)=\frac{\left[\gamma_{p p}^{2} L^{2}-c^{2} m^{2} \pi\right] \Gamma_{1} t}{8 Q_{A} L \gamma_{p p}}+\varphi_{m}
$$

where $D_{m}$ and are $\Omega_{m}$ constants.

Therefore, when the mass effect of the particle is considered, the first approximation to the homogeneous system is given by

$$
\bar{V}(m, t)=D_{m} \operatorname{Cos}\left[\alpha_{m m} t-\Omega_{m}\right]
$$

where

$$
\alpha_{m m}=\gamma_{p p}\left\{1-\frac{\Gamma_{1}}{8}\left(\frac{L^{2} \gamma_{p p}^{2}-c^{2} m^{2} \pi^{2}}{Q_{A} L \gamma_{p p}}\right)\right\}
$$

is the modified natural frequency of the free system due to the presence of the moving partially distributed mass. Thus, to solve the non-homogeneous (29), the differential operator which acts on $\bar{V}(m, t)$ is replaced by the equivalent free system operator defined by the modified frequency $\alpha_{m m}$ namely 
$\bar{V}_{t t}(m, t)+\alpha_{m m}^{2} \bar{V}(m, t)=\frac{\Gamma_{1} L^{2} g}{m \pi Q_{A}}\left[-(-1)^{m}+\cos \frac{m \pi}{L}\left(x_{0}+c t+\frac{1}{2} a t^{2}\right]\right.$

Solving (52) in conjunction with the initial condition, one obtains expression for $\bar{V}(m, t)$. Thus, in view (10)

$$
\begin{aligned}
& \bar{V}(x, t)=\frac{2}{L} \sum_{m=1}^{\infty} \frac{\Gamma_{1} L g \sqrt{\pi}}{2 \alpha_{m m} \sqrt{2 a} Q_{A}}\left\{\operatorname { S i n } \alpha _ { m m } t \left[\operatorname{Sin}\left(\frac{b_{2}^{2}}{4 a}-c_{0}\right) S\left(\frac{b_{2}+2 a t}{\sqrt{2 \pi a}}\right)+C\left(\frac{b_{2}+2 a t}{\sqrt{2 \pi a}}\right) \operatorname{Cos}\left(\frac{b_{2}^{2}}{4 a}-c_{0}\right)\right.\right. \\
& +\operatorname{Sin}\left(\frac{b_{1}^{2}}{4 a}-c_{0}\right) S\left(\frac{b_{1}+2 a t}{\sqrt{2 \pi a}}\right)+C\left(\frac{b_{1}+2 a t}{\sqrt{2 \pi a}}\right) \operatorname{Cos}\left(\frac{b_{1}^{2}}{4 a}-c_{0}\right)-\operatorname{Sin}\left(\frac{b_{2}^{2}}{4 a}-c_{0}\right) S\left(\frac{b_{2}}{\sqrt{2 \pi a}}\right)-C\left(\frac{b_{2}}{\sqrt{2 \pi a}}\right) \operatorname{Cos}\left(\frac{b_{2}^{2}}{4 a}-c_{0}\right) \\
& \left.-\operatorname{Sin}\left(\frac{b_{1}^{2}}{4 a}-c_{0}\right) S\left(\frac{b_{1}}{\sqrt{2 \pi a}}\right)-C\left(\frac{b_{1}}{\sqrt{2 \pi a}}\right) \operatorname{Cos}\left(\frac{b_{1}^{2}}{4 a}-c_{0}\right)-\frac{1}{2 \gamma_{b j}}\left[\operatorname{Sin}\left(m \pi+\alpha_{m n} t\right)-\operatorname{Sin}\left(m \pi-\alpha_{m m} t\right)\right]\right] \\
& -\frac{\operatorname{Cos} \alpha_{m n} t}{\alpha_{m m}}\left[-\operatorname{Sin}\left(\frac{b_{1}^{2}}{4 a}-c_{0}\right) c\left(\frac{b_{1}+2 a t}{\sqrt{2 \pi a}}\right)+S\left(\frac{b_{1}+2 a t}{\sqrt{2 \pi a}}\right) \operatorname{Cos}\left(\frac{b_{1}^{2}}{4 a}-c_{0}\right)+\operatorname{Sin}\left(\frac{b_{2}^{2}}{4 a}-c_{0}\right) C\left(\frac{b_{2}+2 a t}{\sqrt{2 \pi a}}\right)\right. \\
& -S\left(\frac{b_{2}+2 a t}{\sqrt{2 \pi a}}\right) \operatorname{Cos}\left(\frac{b_{2}^{2}}{4 a}-c_{0}\right)+\operatorname{Sin}\left(\frac{b_{1}^{2}}{4 a}-c_{0}\right) c\left(\frac{b_{1}}{\sqrt{2 \pi a}}\right)-S\left(\frac{b_{1}}{\sqrt{2 \pi a}}\right) \operatorname{Cos}\left(\frac{b_{1}^{2}}{4 a}-c_{0}\right)-\operatorname{Sin}\left(\frac{b_{2}^{2}}{4 a}-c_{0}\right) c\left(\frac{b_{2}}{\sqrt{2 \pi a}}\right) \\
& \left.\left.+S\left(\frac{b_{2}}{\sqrt{2 \pi a}}\right) \operatorname{Cos}\left(\frac{b_{2}^{2}}{4 a}-c_{0}\right)+\frac{\operatorname{Cos} m \pi}{\alpha_{m m}}+\frac{1}{2 \alpha_{m m}}\left[\operatorname{Cos}\left(m \pi-\alpha_{m m} t\right)+\operatorname{Cos}\left(m \pi+\alpha_{m m} t\right)\right]\right]\right\}\left(\operatorname{Sin} \frac{m \pi}{L} x\right)
\end{aligned}
$$

Equation (53) represents the transverse-displacement response to a partially distributed mass moving with variable velocity of a simply supported uniform Rayleigh beam resting on Pasternak elastic foundation.

\section{Discussion OF the ANALYTICAl SOlUtions}

When an undamped system such as this is studied, one is interested in the resonance conditions in the dynamical system. This is required because the transverse displacement of an elastic beam may grow without bound which may be catastrophic. Equation (27) clearly shows that the simply supported finite uniform beam resting on elastic foundation and traversed by moving distributed force experiences resonance effect whenever

$\gamma_{p p}=\frac{m \pi c_{c}}{L}, \quad \gamma_{p p}=\frac{m \pi c_{c}}{L}+2 a t_{c}$

while (53) indicates that the same beam under the action of moving distributed mass will experience resonance effect whenever

$\alpha_{m m}=\frac{m \pi c_{c}}{L}, \quad \alpha_{m m}=\frac{m \pi c_{c}}{L}+2 a t_{c}$

where $c_{c}$ and $t_{c}$ are respectively the critical velocity and critical time at which resonance occurs.

From (51), we know that

$$
\alpha_{m m}=\gamma_{p p}\left\{1-\frac{\Gamma_{1}}{8}\left(\frac{L^{2} \gamma_{p p}^{2}-c^{2} m^{2} \pi^{2}}{Q_{A} L \gamma_{p p}}\right)\right\}
$$

which implies $\gamma_{p p}=\frac{\frac{m \pi c_{c}}{L}}{\left\{1-\frac{\Gamma_{1}}{8}\left(\frac{L^{2} \gamma_{p p}^{2}-c^{2} m^{2} \pi^{2}}{Q_{A} L \gamma_{p p}}\right)\right\}}=\frac{m \pi c_{c}}{L}$

From (57), it is evident that for the same natural frequency, the critical velocity for the system of a finite uniform simply supported Rayleigh beam resting on an elastic foundation and traversed by partially distributed forces moving with variable velocity is greater than that of the moving distributed mass problem. Thus, for the same natural frequency of a finite uniform beam, resonance is reached earlier in the moving mass system than in the moving force system.

\section{Numerical CALCUlations AND Discussion}

In this section, calculation of practical interest in dynamics of structures and Engineering design are presented. An elastic uniform Rayleigh beam of length $12.192 m$ has been considered. Furthermore, it is assumed that the modulus of elasticity $E=3.1 \times 10^{10} \mathrm{~N} / \mathrm{m}^{2}$, moment of inertia $I=2.87698 \times 10^{-3} \mathrm{~m}^{4}$, the mass ratio $\varepsilon_{0}$ is 0.25 and the mass per unit length of the beam $\mu$ is $2758.291 \mathrm{~kg} / \mathrm{m}$. The values of the foundation stiffness $\mathrm{K}$ are varied between $0 \mathrm{~N} / \mathrm{m}^{3}$ and $4000000 \mathrm{~N} / \mathrm{m}^{3}$, axial force $\mathrm{N}$ is varied between $0 N$ and $2 \times 10^{6} N$ and shear modulus $\mathrm{G}$ is varied between $0 N / m$ and $3 \times 10^{5} \mathrm{~N} / \mathrm{m}$. The transverse deflections of Rayleigh beam are calculated and plotted against time for various values of foundation stiffness $\mathrm{K}$, axial force $\mathrm{N}$, shear modulus $\mathrm{G}$ and rotatory inertia $\mathrm{R}^{0}$.

Fig. 1 displays the transverse displacement response of uniform Rayleigh beam under the action of partially distributed forces moving at variable velocity for various values of foundation stiffness $\mathrm{K}$ and for fixed values of axial force $N=20000$, shear modulus $G=10000$ and rotatory inertia correction factor $R^{0}=0.5$. The figure shows that the response amplitude of the Rayleigh beam decreases as the values of foundation stiffness increases. On the other hand, Fig. 2 shows the deflection profile of the Rayleigh beam for various values of axial force and fixed values of foundation stiffness $K=40000$, shear modulus $G=10000$ and rotatory inertia correction factor $R^{0}=0.5$. It is observed that higher values of axial force reduce the deflections of the vibrating structure. In Fig. 3, for various time $t$, the displacements of a Rayleigh beam to partially distributed forces travelling at variable velocity for various values of shear modulus $G$ and fixed values of foundation stiffness $K=40000$, axial force $N=20000$ and rotatory inertia correction factor $R^{0}=0.5$ is displayed. The figure clearly shows that the response amplitude of the uniform beam under the action of partially distributed forces travelling at variable velocity decreases with increasing values of shear modulus G. Fig. 4 depicts the transverse displacement response of Rayleigh beam to partially 
distributed forces moving at variable velocity for various values of rotatory inertia correction factor $R^{0}$ and for fixed values of foundation stiffness $K=40000$, axial force $N=20000$ and shear modulus $G=10000$. It is observed that higher values of rotatory inertia correction factor $R^{0}$ reduce the response amplitude of the uniform beam under the action of partially distributed forces. Also, Fig. 5 displays the deflection profile of the Rayleigh beam under moving distributed force for fixed values of axial force $N=20000$, shear modulus $G=10000$ and rotatory inertia correction factor $R^{0}=0.5$ and for various values of foundation stiffness K. Results and analysis similar to Fig. 1 are obtained. Also for various values of axial force, the response amplitude of the Rayleigh beam under moving distributed masses for fixed values of foundation stiffness $K=40000$, shear modulus $G=10000$ and rotatory inertia correction factor $R^{0}=0.5$ is displayed in Fig. 6. Similar results to those of moving distributed force for the uniform Rayleigh beams in Fig. 5 are obtained. Furthermore, Fig. 7 depicts the displacement response of the beam- structure under the action of partially distributed masses travelling at varying velocities for various values of shear modulus $G$ and for fixed values of foundation stiffness $K=40000$, axial force $N=20000$ and rotatory inertia correction factor $R^{0}=0.5$. As in Fig. 3, the displacement response decreases as the values of shear modulus $G$ increases.

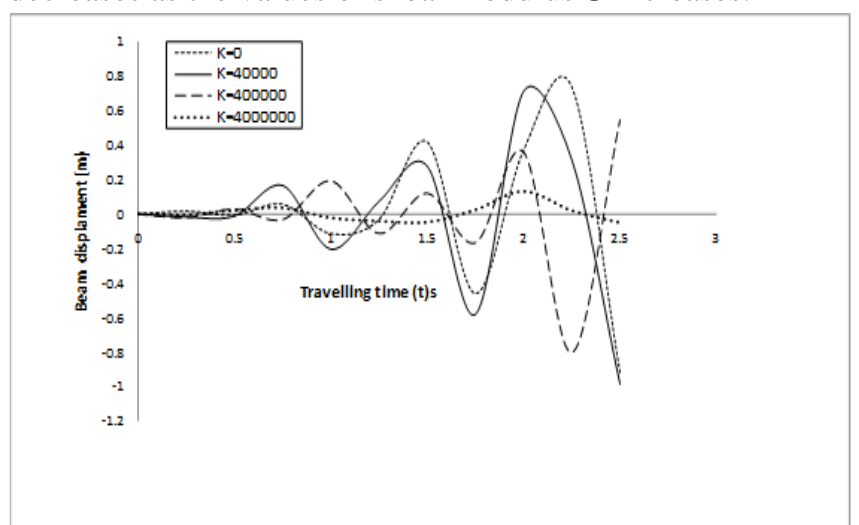

Fig. 1. Transverse displacement of a simply supported uniform Rayleigh beam under the actions of partially distributed forces travelling at variable velocity for various values of foundation stiffness $\mathrm{K}$

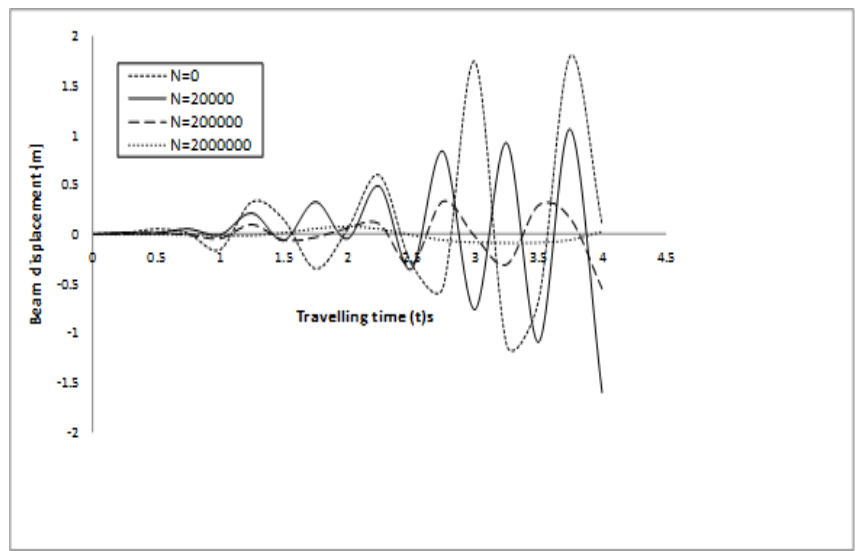

Fig. 2. Deflection profile of a simply supported uniform Rayleigh beam under the actions of partially distributed forces travelling at variable velocity for various values of axial force $\mathrm{N}$

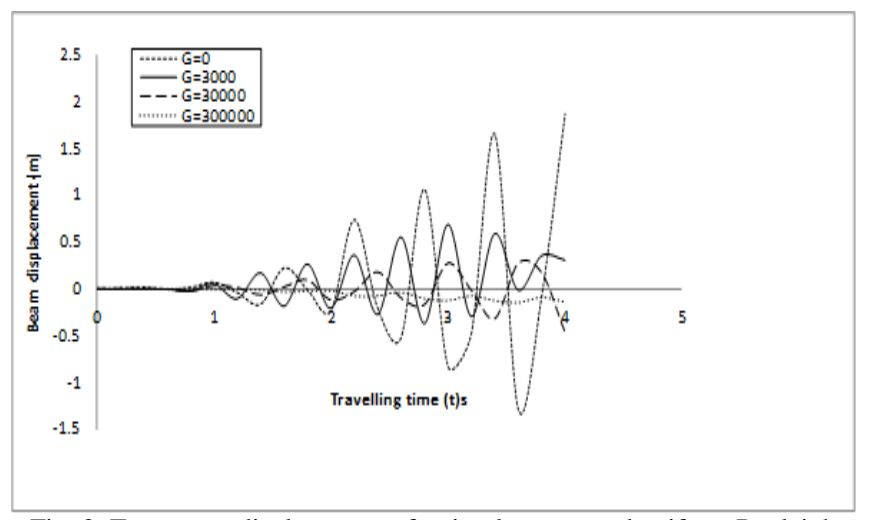

Fig. 3. Transverse displacement of a simply supported uniform Rayleigh beam under the actions of partially distributed forces travelling at variable velocity for various values of shear modulus $G$

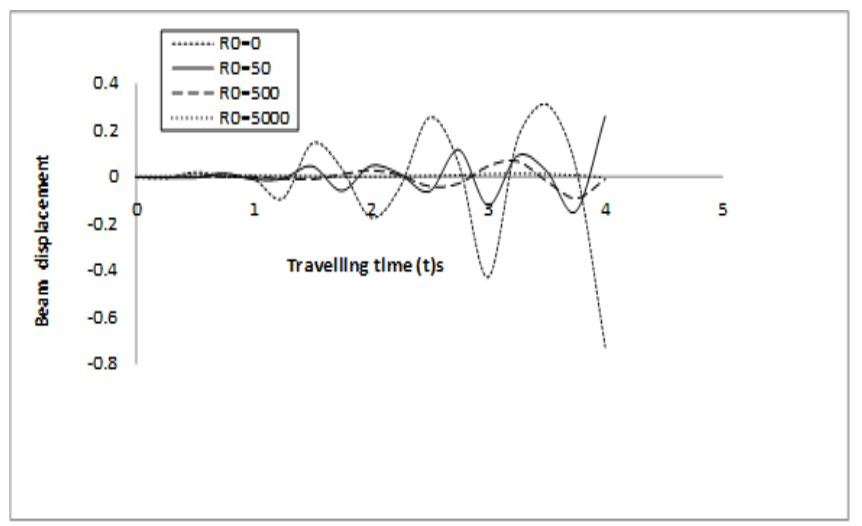

Fig. 4. Response amplitude of a simply supported uniform Rayleigh beam under the actions of partially distributed forces travelling at variable velocity for various values of rotatory inertia correction factor.

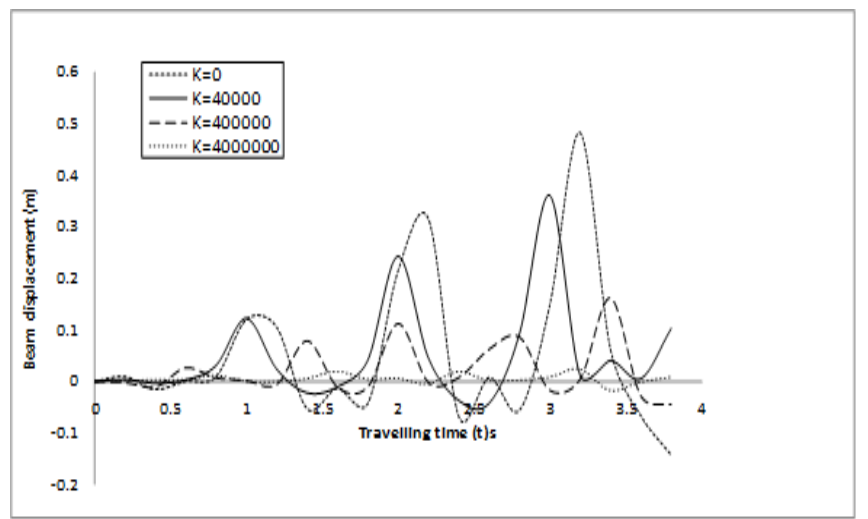

Fig. 5. Transverse displacement of a simply supported uniform Rayleigh beam under the actions of partially distributed masses travelling at variable velocity for various values of foundation stiffness $\mathrm{K}$

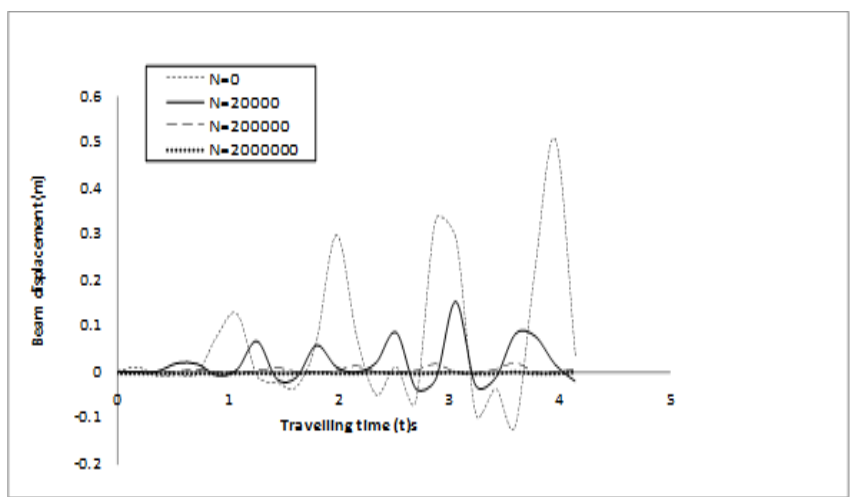

Fig. 6. Deflection profile of a simply supported uniform Rayleigh beam under the actions of partially distributed masses travelling at variable velocity for various values of axial force $\mathrm{N}$ 


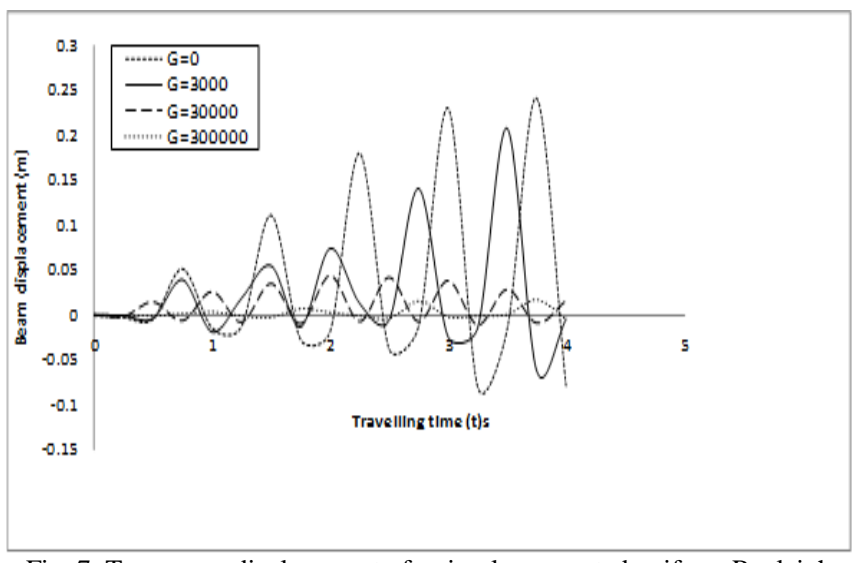

Fig. 7. Transverse displacement of a simply supported uniform Rayleigh beam under the actions of partially distributed masses travelling at variable velocity for various values of shear modulus $G$

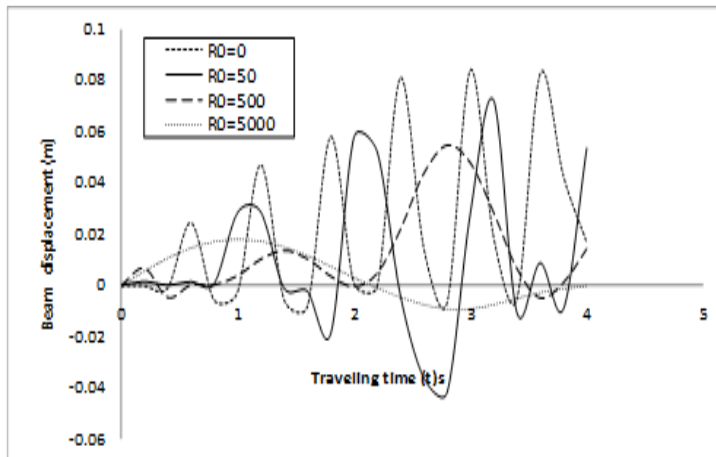

Fig. 8. Response amplitude of a simply supported uniform Rayleigh beam under the actions of partially distributed masses travelling at variable velocity for various values of rotatory inertia correction factor

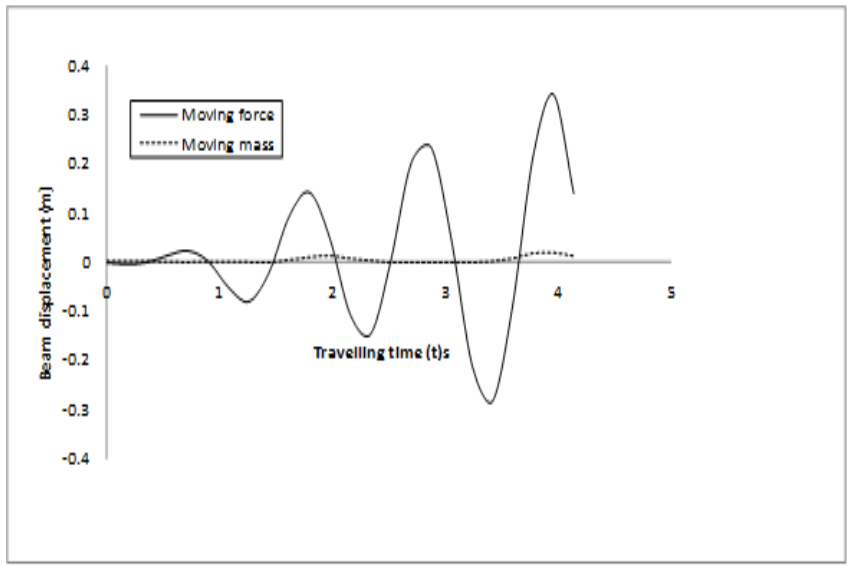

Fig. 9. Comparison of the displacement response of moving force and moving mass cases for a uniform simply supported Rayleigh beam for fixed

$$
\text { values of } K, N, G \text {, and } R^{0}
$$

The transverse displacement of the beam under the action of partially distributed masses for fixed values of foundation stiffness $K=40000$, axial force $N=20000$ and shear modulus $G=10000$ for various values of rotatory inertia correction factor $R^{0}$ is shown in Fig. 8. It is evident, as $R^{0}$ increases, the transverse displacements decrease. Finally, Fig. 9 shows the comparison of the transverse displacement response of moving force and moving mass cases for simply supported uniform Rayleigh beam traversed by a moving load travelling at variable velocity for $K=400000$,
$N=200000, G=100000$ and $R^{0}=0.5$. Clearly, the response amplitude of the moving distributed force is higher than that of moving distributed mass. This important result has also been reported in Andi, Oni and Ogunbamike [18] and Oni and Awodola [19] for the cases when the travelling load is moving at constant velocity.

\section{CONCLUSION}

The problem of a uniform simply supported Rayleigh beam under moving partially distributed load is investigated in this paper. The effects of gravity and inertia of the distributed loads are taken into consideration. The fourth order partial differential equation governing the motion of the beam was solved using the generalized finite integral transform and the modified asymptotic method of Struble. Numerical analysis is carried out and results show that the transverse displacements of both the moving partially distributed force and moving partially distributed mass problems decrease with increase in the values of foundation stiffness and shear modulus. Similarly, higher values of axial force and rotatory inertia correction factor reduce the response amplitudes of both moving partially distributed force and moving partially distributed mass problems. Furthermore, it is observed that for the uniform Rayleigh beam having simple supports at both ends, for fixed values of foundation moduli, axial force, shear modulus and rotatory inertia correction factor, the transverse deflection under the actions of moving partially distributed masses is higher than that under the actions of moving partially distributed force. Thus, moving distributed force solution is not always an upper bound to moving partially distributed mass problems and hence the inertia effect of the moving distributed load must be considered for accurate and safe assessment of the response to moving distributed load of elastic structural members. Finally, the critical speed for the system traversed by a moving distributed force is found to be greater than that under the influence of moving distributed mass showing that resonance is reached earlier in moving distributed mass problem.

\section{REFERENCES}

[1] L. Fryba,. Vibration of solids and structures under moving load, Noordhoff International Publishing Groningen, The Netherlands, 1972.

[2] M.R. Shadnan, F.R. Rofooei, F, Mofid, and B. Mehri, "Periodicity in the response of non-linear plate under moving mass," Thin-walled Structures, 40: 283-295, 2002.

[3] E. Savin, "Dynamic amplification factor and response spectrum for the evaluation of vibrations of beams under successive moving loads," Journal of sound and vibration, 248(2): 267-288, 2001.

[4] J. A. Gbadeyan, and Y.M. Aiyesimi,. "On the dynamic analysis of elastic beam and plate resting on viscoelastic foundation to load moving at non-uniform speed," Nigerian Journal of Mathematics and Applications, 3: 73-90, 1990.

[5] S.S. Kokhmanyuk and A. P. Filippov, "Dynamic effects on a beam of a load moving at variable speed," Stroitel' n mekhanka I raschet sooruzhenii, 9(2): 36-39, 1967.

[6] S.T. Oni, "Flexural motions of uniform beam under the actions of concentrated mass travelling with variable velocity," Journal of the Nigerian Association of Mathematical Physics, 8: 215-224, 2004.

[7] M. H. Huang. and D. P. Thambiratnam, "Deflection response of plate on Winkler foundation to moving accelerated loads," Engineering structures, 23: 1134-1141, 2001. 
[8] S. T. Oni and B. Omolofe, "Dynamic analysis of a prestressed elastic beam with general boundary conditions under moving loads at varying velocities," Journal of Engineering and Engineering Technology, FUTA, vol. 4(1): 55-72, 2005.

[9] S. T. Oni and B. Omolofe, "Flexural motions under accelerated loads of structurally prestressed beam with general boundary conditions resting on elastic foundation," Latin American Journal of Solids and Structures, 7(3): 285-309, 2010.

[10] Alaa Tawfiq Ahmed (2011)." Simulation of transient dynamic loads on beams with variable velocities," European Journal of Scientific Research, 62(4): 481-490, 2011.

[11] Ismail Esen, "Dynamic response of a beam due to an accelerating moving mass using finite element approximation," Mathematical and Computation Applications, 16(1): 171-182, 2011.

[12] Mingliang Li., Tao Qian., Yang Zhong and Hua Zheng, "Dynamic response of the rectangular plate subjected to moving loads with variable velocity," Journal of Engineering Mechanics, 140: DOI:10.106/0000687 (ASME) EM 1943-7889, 2014.

[13] J. A. Gbadeyan. and S. T. Oni, "Dynamic behaviour of beams and rectangular plates under moving loads," Journal of Sound and Vibration, 182(5): 677-695, 1995.

[14] S.T. Oni and B. Omolofe, "Dynamic response of prestressed Rayleigh beam prestressed Rayleigh beam resting on elastic foundation and subjected to masses travelling at varying velocity," Journal of Vibration and Acoustics, 133: 041005-1-15, 2011.

[15] L. Sun, "Dynamic displacement response of beam-type structures to moving loads," International Journal of Solids and Structures, 38(3839): 8869-8878, 2001.

[16] Y. M. Wang, "The dynamic analysis of a finite inextensible beam with an attached accelerating mass," International Journal of Solids and Structures, (9-19): 831-854, 1998.

[17] S. Sadiku. and H. H. E. Leipholz, "On the dynamics of elastic systems with moving concentrated masses," Ingenieur Achieves, 57: 223-242, 1989.

[18] E. A. Andi, S. T. Oni and O. K. Ogunbamike, "Dynamic analysis of a finite simply supported uniform Rayleigh beam under travelling distributed loads," Journal of the Nigerian Association of Mathematical Physics, 26: 125-136, 2014.

[19] S. T. Oni. and T. O. Awodola, "Dynamic behaviour under moving concentrated masses of elastically supported finite Bernoulli-Euler beam on Winkler elastic foundation," Latin American Journal of Solids and Structures. 7(3): 285-309, 2010.

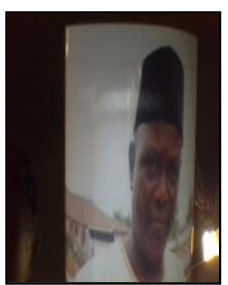

Oni, S.T. was born in Ondo State, Nigeria about six decades ago. He holds a Bachelor of Science, Master of Science and Doctor of Philosophy degrees from the Department of Mathematics, University of Ilorin, Ilorin Nigeria. He is currently a Professor of Applied Mathematics in the Department of Mathematical Science, Federal University of Technology, Akure and has over sixty publications and conference proceedings to his credit. Prof. Oni is a registered member of American Mathematical Society, Nigerian Mathematical Society, External Assessor to various universities within and abroad and also a reviewer to various local and international journals. Email: sundayoni1958@yahoo.ca.

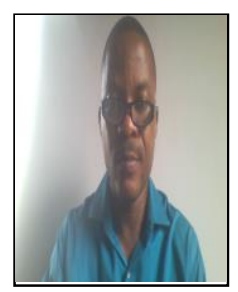

Ogunbamike, O. K. was born in Ondo State, Nigeria about five decades ago. He graduated with a Bachelor of Technology degree in Industrial Mathematics in 1997 and a Master of Technology in Applied Mathematics in 2008 from The Federal University of Technology, Akure, Nigeria. He then completed his Doctor of Philosophy programme from the same university in 2014. He is currently an academic staff in the Department of Mathematical Sciences, Ondo State University of Science and Technology, Okitipupa, Nigeria. His area of specialization is Dynamics of Structures under moving load. Dr. Ogunbamike has numerous publications in reputable journals and is a member of some learned societies. Email: ogunbamike2005@gmail.com 